\title{
Power loss reduction by gryllidae optimization algorithm
}

\author{
Kanagasabai Lenin \\ Department of EEE, Prasad V. Potluri Siddhartha Institute of Technology, India
}

\begin{tabular}{l} 
Article Info \\
\hline Article history: \\
Received Nov 12, 2019 \\
Revised Jan 20, 2020 \\
Accepted Feb 19, 2020 \\
\hline
\end{tabular}

Keywords:

Gryllidae optimization algorithm,

Optimal reactive power,

Transmission loss,

\begin{abstract}
This paper projects Gryllidae Optimization Algorithm (GOA) has been applied to solve optimal reactive power problem. Proposed GOA approach is based on the chirping characteristics of Gryllidae. In common, male Gryllidae chirp, on the other hand some female Gryllidae also do as well. Male Gryllidae draw the females by this sound which they produce. Moreover, they caution the other Gryllidae against dangers with this sound. The hearing organs of the Gryllidae are housed in an expansion of their forelegs. Through this, they bias to the produced fluttering sounds. Proposed Gryllidae Optimization Algorithm (GOA) has been tested in standard IEEE 14,30 bus test systems and simulation results show that the projected algorithms reduced the real power loss considerably.
\end{abstract}

This is an open access article under the CC BY-SA license.

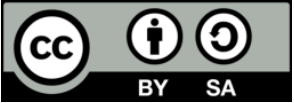

\section{Corresponding Author:}

Kanagasabai Lenin,

Department of EEE,

Prasad V. Potluri Siddhartha Institute of Technology,

Kanuru, Vijayawada, Andhra Pradesh, 520007, India.

Email: gklenin@gmail.com

\section{INTRODUCTION}

Reactive power problem plays a key role in secure and economic operations of power system. Optimal reactive power problem has been solved by variety of types of methods [1-6]. Nevertheless numerous scientific difficulties are found while solving problem due to an assortment of constraints. Evolutionary techniques [7-17] are applied to solve the reactive power problem, but the main problem is many algorithms get stuck in local optimal solution \& failed to balance the Exploration \& Exploitation during the search of global solution. This paper projects Gryllidae Optimization Algorithm (GOA) has been applied to solve optimal reactive power problem. Proposed algorithm based on the chirping characteristics of Gryllidae and formulated to solve the optimal reactive power problem. In common, male Gryllidae chirp, on the other hand some female Gryllidae also do as well. Male Gryllidae draw the females by this sound which they produce. Male Gryllidae create this sound by chirping the wings and known as stridulating. They attract each other by this sound for mating and keep the others from their nests. Moreover, they caution the other Gryllidae against dangers with this sound. The hearing organs of the Gryllidae are housed in an expansion of their forelegs. Through this, they bias to the produced fluttering sounds. Proposed Gryllidae Optimization Algorithm (GOA) has been tested in standard IEEE 14, 30 bus test systems and simulation results show that the projected algorithms reduced the real power loss considerably.

\section{PROBLEM FORMULATION}

Objective of the problem is to reduce the true power loss:

$\mathrm{F}=\mathrm{P}_{\mathrm{L}}=\sum_{\mathrm{k} \in \mathrm{Nbr}} \mathrm{g}_{\mathrm{k}}\left(\mathrm{V}_{\mathrm{i}}^{2}+\mathrm{V}_{\mathrm{j}}^{2}-2 \mathrm{~V}_{\mathrm{i}} \mathrm{V}_{\mathrm{j}} \cos \theta_{\mathrm{ij}}\right)$ 
voltage deviation given as follows:

$$
\mathrm{F}=\mathrm{P}_{\mathrm{L}}+\omega_{\mathrm{v}} \times \text { Voltage Deviation }
$$

voltage deviation given by:

$$
\text { Voltage Deviation }=\sum_{\mathrm{i}=1}^{\mathrm{Npq}}\left|\mathrm{V}_{\mathrm{i}}-1\right|
$$

constraint (equality)

$$
P_{G}=P_{D}+P_{L}
$$

constraints (Inequality)

$$
\begin{aligned}
& \mathrm{P}_{\text {gslack }}^{\min } \leq \mathrm{P}_{\text {gslack }} \leq \mathrm{P}_{\text {gslack }}^{\text {max }} \\
& \mathrm{Q}_{\mathrm{gi}}^{\min } \leq \mathrm{Q}_{\mathrm{gi}} \leq \mathrm{Q}_{\mathrm{gi}}^{\max }, \mathrm{i} \in \mathrm{N}_{\mathrm{g}} \\
& \mathrm{V}_{\mathrm{i}}^{\mathrm{min}} \leq \mathrm{V}_{\mathrm{i}} \leq \mathrm{V}_{\mathrm{i}}^{\mathrm{max}}, \mathrm{i} \in \mathrm{N} \\
& \mathrm{T}_{\mathrm{i}}^{\min } \leq \mathrm{T}_{\mathrm{i}} \leq \mathrm{T}_{\mathrm{i}}^{\text {max }}, \mathrm{i} \in \mathrm{N}_{\mathrm{T}} \\
& \mathrm{Q}_{\mathrm{c}}^{\min } \leq \mathrm{Q}_{\mathrm{c}} \leq \mathrm{Q}_{\mathrm{C}}^{\max }, \mathrm{i} \in \mathrm{N}_{\mathrm{C}}
\end{aligned}
$$

\section{GRYLLIDAE OPTIMIZATION ALGORITHM}

Gryllidae optimization algorithm based on the chirping characteristics of Gryllidae and formulated to solve the optimal reactive power problem. In common, male Gryllidae chirp, on the other hand some female Gryllidae also do as well. Male Gryllidae draw the females by this sound which they produce. Male Gryllidae create this sound by chirping the wings and known as stridulating. They attract each other by this sound for mating and keep the others from their nests. Moreover, they caution the other Gryllidae against dangers with this sound. The hearing organs of the Gryllidae are housed in an expansion of their forelegs. Through this, they bias to the produced fluttering sounds. Temperature $\left(T_{f}\right)$ in degrees Fahrenheit is calculated from the chirp $\left(\mathrm{N}_{\mathrm{c}}\right.$ (per minute)) by,

$$
T_{f}=50.00+\frac{\text { Number of chrips }\left(N_{c}\right)-40.00}{4.00}
$$

temperature in degrees Celsius $\left(\mathrm{T}_{\mathrm{c}}\right)$, is defined by,

$$
T_{c}=10.00+\frac{\text { Number of chrips }\left(N_{c}\right)-40.00}{7.00}
$$

chirping rate can be calculated based on $T_{c}$ and $T_{f}$ is found by,

$$
\begin{aligned}
& N_{c}=\left(T_{c}-10.00\right) * 7.00+40.00 \\
& N_{c}=\left(T_{f}-50.00\right) * 4.00+40.00
\end{aligned}
$$

velocity of sound is calculated by via the temperature attained with reference to the Dolbear law

$$
V=20.1 * \sqrt{273+C}
$$

frequency of the sound is given by,

$$
f=\frac{V}{\lambda}
$$


intensity of the sound inversely proportional to the square of the distance,

$$
I=\frac{W}{4 \pi r^{2}}=\frac{p}{\rho c} ; W=I * 4 \pi r^{2}
$$

sound propagation level found by,

$$
L_{p}=L_{w}+10^{*} \log \left[Q / 4 \pi r^{2}\right]
$$

atmosphere sound absorption is given by,

$$
A_{\text {atmos }}=7.4\left(f^{2} r / \Phi\right) 10^{-8}
$$

free filed sound pressure is given by,

$$
L_{p}^{\prime}=L_{p}-A_{\text {atmos }}
$$

frequency, velocity, position value obtained by,

$$
\begin{aligned}
& f_{i}=f_{\text {minimum }}+\left(f_{\text {maximum }}-f_{\text {mimimum }}\right) \beta \\
& v_{i}^{t}=v_{i}^{t-1}+\left(x_{i}-x_{*}\right) f_{i}+V_{i} \\
& x_{i}^{t}=x_{i}^{t-1}+v_{i}^{t}
\end{aligned}
$$

random walk [18] is done through,

$$
x_{i}=x_{\text {best }}+0.01 * \text { random }(0,1)
$$

in the period of the modernizing procedure, Euclidian distances ( $r$ ) among all of the Gryllidae in the population were computed by,

$$
\begin{aligned}
& K=K o e^{-\gamma r^{2}} \\
& x_{i}=x_{i}+K o e^{-\gamma r_{i j}^{2}}+\alpha \epsilon_{i}
\end{aligned}
$$

Commence

For $\mathrm{i}=1$ to $\mathrm{n}$ do

$x_{i} \leftarrow$ engender initial solution ( )

End

$i_{\text {min }} \leftarrow \operatorname{argmin}_{i} s\left(x_{i}\right)$

$F_{\min } \leftarrow \operatorname{argmin} s\left(x_{i}\right)$

$x_{i} \min \leftarrow \operatorname{argmin}_{x i} s\left(x_{i}\right)$

For $\mathrm{i}=1$ to $\mathrm{t}$ do

while $\left(F_{\text {minimum }}>\right.$ Tol $)$

For $\mathrm{i}=1$ to $\mathrm{n}$ do

$N_{i} \leftarrow$ engender random vector

$T_{i} \leftarrow$ Dolbear law $\left(N_{i}\right)$

$c_{i}=(5 / 9) T_{i}-32$

$V_{i} \leftarrow V=20.1^{*} \sqrt{273+C}, \lambda \leftarrow x_{i}-x_{\text {best }}$

$F_{\text {maximum }} \leftarrow f=\frac{V}{\lambda}, f_{i}, v_{i}^{t}, x_{i}^{t} \leftarrow f_{i}=f_{\text {minimum }}+\left(f_{\text {maximum }}-f_{\text {mimimum }}\right) \beta, \quad v_{i}^{t}=v_{i}^{t-1}+\left(x_{i}-x_{*}\right) f_{i}+$ $V_{i}, x_{i}^{t}=x_{i}^{t-1}+v_{i}^{t}$

$\gamma \leftarrow$ compute the Coefficient

if random $[0,1]>\gamma$

For $\mathrm{j}=1$ to $\mathrm{n}$ do

if $s_{i}<s_{j}$ then $r_{j} \leftarrow K=K o e^{-\gamma r^{2}}$

$P S \leftarrow I=\frac{W}{4 \pi r^{2}}=\frac{p}{\rho c} ; W=I * 4 \pi r^{2}$

$L P \leftarrow L_{p}=L_{w}+10^{*} \log \left[Q / 4 \pi r^{2}\right]$

$A_{\text {atmos }} \leftarrow A_{\text {atmos }}=7.4\left(f^{2} r / \Phi\right) 10^{-8}$ 
$R L_{p} \leftarrow L_{p}^{\prime}=L_{p}-A_{\text {atmos }}$

$K_{0} \leftarrow R L_{p}, K_{i} \leftarrow x_{i}=x_{i}+K o e^{-\gamma r_{i j}^{2}}+\alpha \epsilon_{i}$

$x_{i} \leftarrow x_{i}=x_{\text {best }}+0.01 *$ random $(0,1)$

End

$F_{\text {new }} \leftarrow \operatorname{cost}$ function $\left(x_{i}\right)$

if $\left(F_{\text {new }}<F_{\text {minimum }}\right)$

$x_{\text {best }}=x_{i}$

$F_{\text {minimum }}=F_{\text {new }}$

End

Find the most excellent solution

End

\section{SIMULATION RESULTS}

At first in standard IEEE 14 bus system the validity of the proposed Gryllidae Optimization Algorithm (GOA) has been tested, Table 1 shows the constraints of control variables Table 2 shows the limits of reactive power generators and comparison results are presented in Table 3.

Then the proposed Gryllidae Optimization Algorithm (GOA) simulated in IEEE 30 Bus system. Table 4 shows the constraints of control variables, Table 5 shows the limits of reactive power generators and comparison results are presented in Table 6.

Table 1. Constraints of control variables

\begin{tabular}{cccc}
\hline System & Variables & Minimum (PU) & Maximum (PU) \\
\hline IEEE 14 Bus & Generator Voltage & 0.95 & 1.1 \\
& Transformer Tap & 0.9 & 1.1 \\
& VAR Source & 0 & 0.20 \\
\hline
\end{tabular}

Table 2. Constrains of reactive power generators

\begin{tabular}{cccc}
\hline System & Variables & Q Minimum (PU) & Q Maximum (PU) \\
IEEE 14 Bus & 1 & 0 & 10 \\
\hline & 2 & -40 & 50 \\
& 3 & 0 & 40 \\
& 6 & -6 & 24 \\
& 8 & -6 & 24 \\
\hline
\end{tabular}

Table 3. Simulation results of IEEE -14 system

\begin{tabular}{|c|c|c|c|c|c|c|}
\hline $\begin{array}{c}\text { Control } \\
\text { variables }\end{array}$ & Base case & MPSO [19] & PSO [19] & EP [19] & SARGA [19] & GOA \\
\hline$V G-1$ & 1.060 & 1.100 & 1.100 & $\mathrm{NR}^{*}$ & $\mathrm{NR}^{*}$ & 1.004 \\
\hline$V G-2$ & 1.045 & 1.085 & 1.086 & 1.029 & 1.060 & 1.014 \\
\hline$V G-3$ & 1.010 & 1.055 & 1.056 & 1.016 & 1.036 & 1.009 \\
\hline$V G-6$ & 1.070 & 1.069 & 1.067 & 1.097 & 1.099 & 1.002 \\
\hline$V G-8$ & 1.090 & 1.074 & 1.060 & 1.053 & 1.078 & 1.011 \\
\hline Tap 8 & 0.978 & 1.018 & 1.019 & 1.04 & 0.95 & 0.900 \\
\hline Tap 9 & 0.969 & 0.975 & 0.988 & 0.94 & 0.95 & 0.911 \\
\hline Tap 10 & 0.932 & 1.024 & 1.008 & 1.03 & 0.96 & 0.901 \\
\hline$Q C-9$ & 0.19 & 14.64 & 0.185 & 0.18 & 0.06 & 0.124 \\
\hline$P G$ & 272.39 & 271.32 & 271.32 & $\mathrm{NR}^{*}$ & NR* & 271.46 \\
\hline$Q G$ (Mvar) & 82.44 & 75.79 & 76.79 & $\mathrm{NR}^{*}$ & NR* & 75.04 \\
\hline $\begin{array}{l}\text { Reduction in } \\
\text { PLoss }(\%)\end{array}$ & 0 & 9.2 & 9.1 & 1.5 & 2.5 & 12.33 \\
\hline $\begin{array}{l}\text { Total PLoss } \\
(\mathrm{Mw})\end{array}$ & 13.550 & 12.293 & 12.315 & 13.346 & 13.216 & 11.879 \\
\hline
\end{tabular}

Table 4. Constraints of control variables

\begin{tabular}{llll}
\hline System & Variables & Minimum $(\mathrm{PU})$ & Maximum $(\mathrm{PU})$ \\
\hline IEEE 30 Bus & Generator Voltage & 0.95 & 1.1 \\
& Transformer Tap & 0.9 & 1.1 \\
& VAR Source & 0 & 0.20 \\
\hline
\end{tabular}

Int J Inf \& Commun Technol, Vol. 9, No. 3, December 2020: 179 - 184 
Table 5. Constrains of reactive power generators

\begin{tabular}{cccc}
\hline System & Variables & Q Minimum (PU) & Q Maximum $(\mathrm{PU})$ \\
\hline IEEE 30 Bus & 1 & 0 & 10 \\
& 2 & -40 & 50 \\
& 5 & -40 & 40 \\
& 8 & -10 & 40 \\
& 11 & -6 & 24 \\
& 13 & -6 & 24 \\
\hline
\end{tabular}

Table 6. Simulation results of IEEE -30 system

\begin{tabular}{|c|c|c|c|c|c|c|}
\hline $\begin{array}{c}\text { Control } \\
\text { variables }\end{array}$ & Base case & MPSO [19] & PSO [19] & EP [19] & SARGA [19] & GOA \\
\hline$V G-1$ & 1.060 & 1.101 & 1.100 & NR* & $\mathrm{NR}^{*}$ & 1.010 \\
\hline$V G-2$ & 1.045 & 1.086 & 1.072 & 1.097 & 1.094 & 1.008 \\
\hline$V G-5$ & 1.010 & 1.047 & 1.038 & 1.049 & 1.053 & 1.012 \\
\hline$V G-8$ & 1.010 & 1.057 & 1.048 & 1.033 & 1.059 & 1.049 \\
\hline$V G-12$ & 1.082 & 1.048 & 1.058 & 1.092 & 1.099 & 1.032 \\
\hline VG-13 & 1.071 & 1.068 & 1.080 & 1.091 & 1.099 & 1.023 \\
\hline Tap11 & 0.978 & 0.983 & 0.987 & 1.01 & 0.99 & 0.912 \\
\hline Tap12 & 0.969 & 1.023 & 1.015 & 1.03 & 1.03 & 0.905 \\
\hline Tap15 & 0.932 & 1.020 & 1.020 & 1.07 & 0.98 & 0.914 \\
\hline Tap36 & 0.968 & 0.988 & 1.012 & 0.99 & 0.96 & 0.906 \\
\hline QC10 & 0.19 & 0.077 & 0.077 & 0.19 & 0.19 & 0.089 \\
\hline QC24 & 0.043 & 0.119 & 0.128 & 0.04 & 0.04 & 0.101 \\
\hline$P G(\mathrm{MW})$ & 300.9 & 299.54 & 299.54 & $\mathrm{NR}^{*}$ & $\mathrm{NR}^{*}$ & 298.59 \\
\hline$Q G$ (Mvar) & 133.9 & 130.83 & 130.94 & $\mathrm{NR}^{*}$ & $\mathrm{NR}^{*}$ & 131.81 \\
\hline $\begin{array}{l}\text { Reduction in } \\
\text { PLoss }(\%)\end{array}$ & 0 & 8.4 & 7.4 & 6.6 & 8.3 & 10.05 \\
\hline $\begin{array}{c}\text { Total PLoss } \\
(\mathrm{Mw})\end{array}$ & 17.55 & 16.07 & 16.25 & 16.38 & 16.09 & 15.786 \\
\hline
\end{tabular}

\section{Conclusion}

In this paper Gryllidae Optimization Algorithm (GOA) successfully solved the optimal reactive power problem. Application of the Gryllidae Optimization Algorithm is developed by the inspiration of a type of insect; on the recognizable global engineering problems in the simulation-based nature which has recently participated to the meta-heuristic algorithm approach was confirmed. Proposed Gryllidae Optimization Algorithm (GOA) has been tested in standard IEEE 14, 30 bus test systems and simulation results show that the projected algorithms reduced the real power loss considerably.

\section{REFERENCES}

[1] K. Y. Lee, "Fuel-cost minimisation for both real and reactive-power dispatches," Proceedings Generation, Transmission and Distribution Conference, vol. 131, no. 3, pp. 85-93, 1984.

[2] N. I. Deeb, "An efficient technique for reactive power dispatch using a revised linear programming approach," Electric Power System Research, vol. 15, no. 2, pp. 121-134, 1998.

[3] M. R. Bjelogrlic, M. S. Calovic, B. S. Babic, "Application of Newton's optimal power flow in voltage/reactive power control," IEEE Trans Power System, vol. 5, no. 4, pp. 1447-1454, 1990.

[4] S. Granville, "Optimal reactive dispatch through interior point methods," IEEE Transactions on Power System, vol. 9, no. 1, pp. 136-146, 1994.

[5] N. Grudinin, "Reactive power optimization using successive quadratic programming method," IEEE Transactions on Power System, vol. 13, no. 4, pp. 1219-1225, 1998.

[6] Ng Shin Mei, R.; Sulaiman, M.H.; Mustaffa, Z.; Daniyal, H. "Optimal reactive power dispatch solution by loss minimization using moth-flame optimization technique," Appl. Soft Comput., vol. 59, pp. 210-222, 2017.

[7] Chen, G., Liu, L., Zhang, Z., Huang, S. "Optimal reactive power dispatch by improved GSA-based algorithm with the novel strategies to handle constraints," Appl. Soft Comput., vol. 50, pp. 58-70, 2017.

[8] Naderi, E., Narimani, H., Fathi, M., Narimani, M.R, "A novel fuzzy adaptive configuration of particle swarm optimization to solve large-scale optimal reactive power dispatch," Appl. Soft Comput., vol. 53, pp. 441-456, 2017.

[9] Heidari, A.A, Ali Abbaspour, R. Rezaee Jordehi, A. "Gaussian bare-bones water cycle algorithm for optimal reactive power dispatch in electrical power systems," Appl. Soft Comput, vol. 57, pp. 657-671, 2017. 
[10] Mahaletchumi Morgan, Nor Rul Hasma Abdullah, Mohd Herwan Sulaiman, Mahfuzah Mustafa, Rosdiyana Samad, "Benchmark Studies on Optimal Reactive Power Dispatch (ORPD) Based Multiobjective Evolutionary Programming (MOEP) Using Mutation Based on Adaptive Mutation Adapter (AMO) and Polynomial Mutation Operator (PMO)," Journal of Electrical Systems, vol. 12, no. 1, pp. 121-132, 2016

[11] Rebecca Ng Shin Mei, Mohd Herwan Sulaiman, Zuriani Mustaffa, "Ant Lion Optimizer for Optimal Reactive Power Dispatch Solution," Journal of Electrical Systems, Special Issue AMPE2015, pp. 68-74, 2016.

[12] P. Anbarasan, T. Jayabarathi, "Optimal reactive power dispatch problem solved by symbiotic organism search algorithm," Innovations in Power and Advanced Computing Technologies, 2017.

[13] Gagliano A., Nocera F, "Analysis of the performances of electric energy storage in residential applications," International Journal of Heat and Technology, vol. 35, no. 1, pp. S41-S48, 2017.

[14] Caldera M., Ungaro P., Cammarata G., Puglisi G, "Survey-based analysis of the electrical energy demand in Italian households," Mathematical Modelling of Engineering Problems, Vol. 5, No. 3, pp. 217-224, 2018.

[15] M. Basu, "Quasi-oppositional differential evolution for optimal reactive power dispatch," Electrical Power and Energy Systems, vol. 78, pp. 29-40, 2016.

[16] Jonti Deuri, S. Siva Sathya, "A Novel Cricket Chirping Algorithm for Engineering Optimization Problem," Advances in Natural and Applied Sciences, vol. 9, no. 6, pp, 397-402, Special 2015.

[17] A. Kaveh, N. Farhoudi, "A new optimization method: Dolphin echolocation," Advances in Engineering Software, vol. 59, pp. 53-70, 2013.

[18] IEEE, “The IEEE-test systems", http://www.ee.washington.edu/trsearch/pstca/, 1993.

[19] Ali Nasser Hussain, Ali Abdulabbas Abdullah and Omar Muhammed Neda, "Modified Particle Swarm Optimization for Solution of Reactive Power Dispatch," Research Journal of Applied Sciences, Engineering and Technology, vol. 15, no. 8, pp. 316-327, 2018. 